\section{Testing models of the redundant-signals effect: A warning concerning the combination-rule regression analysis}

\section{J. TOBY MORDKOFF \\ University of California-San Diego, La Jolla, California}

The redundant-signals effect is the observed $R T$ advantage for trials presenting two or more targets, as compared with trials with only one target. Two general classes of parallel-processing model have been proposed to explain this effect: race models (e.g., Raab, 1962) and coactivation models (e.g., Miller, 1982). Various distributional analyses have been used in work aimed at discriminating between these two model classes. The present study reexamined one of these tests-the combination-rule regression analysis based on variable-criterion theory (Grice, Canham, \& Boroughs, 1984)-by applying it to the data from two sets of simulated experiments. One set of simulations assumed coactivation; the other set assumed an independent race on redundant-target trials. Nearly identical combination-rule values were observed in the two sets of simulations. This finding shows that the combination rule of variable-criterion theory does not discriminate between models capable of explaining the redundant-signals effect. The implications of this finding are briefly discussed.

When subjects are presented with two targets that both call for the same response, responses are faster (and more accurate) than when only one target is presented (e.g., Egeth \& Mordkoff, 1991; Grice, Canham, \& Boroughs, 1984; Miller, 1982; Mordkoff \& Yantis, 1991; van der Heijden, La Heij, \& Boer, 1983; van der Heijden, Schreuder, Maris, \& Neerincx, 1984). This empirical result is known as a redundancy gain or the redundantsignals effect (RSE), because the second target in the display is redundant in the sense that it does not need to be processed in order to determine which response is correct.

The early work examining the RSE employed mean response time (RT) as the dependent variable (e.g., Biederman \& Checkosky, 1970; Egeth, 1966; Holmgren, Joula, \& Atkinson, 1974; van der Heijden, 1975). Under this analysis, redundant-target mean RT is compared with single-target mean RT by $t$ test. A significant RSE, when found, is then interpreted as evidence of the parallel processing of both targets (for technical details relating to

This work was supported by Public Health Service Training Grant T32-MH14268. The central finding of this study was first noted in the analysis of the smaller set of simulations reported by Egeth and Mordkoff (1991). Thanks are due Howard Egeth, Robert Grice, Cathleen Moore, two anonymous reviewers, and (especially) Jeff Miller for their advice and comments. Correspondence should be sent to J. T. Mordkoff, Center for Human Information Processing, University of California-San Diego, La Jolla, CA 92093-0109 (e-mail: furball@ucsd.edu).
mean-RT analyses, see Miller \& Lopes, 1988; Mullin, Egeth, \& Mordkoff, 1988). To summarize this work: Significant RSEs have been found in a variety of situations, lending support to parallel-processing models.

\section{Models of the Redundant-Signals Effect}

More recent work has attempted to discriminate between two specific classes of parallel-processing models (see Miller, 1982, or Mordkoff \& Yantis, 1991, for an introduction). Members of the first class, known as race models, assume that the two items in each display are encoded and identified separately and in parallel (e.g., Mordkoff \& Yantis, 1991; Raab, 1962; Shaw, 1982; see also Colonius, 1990). This class explains the RSE in terms of statistical facilitation or probability summation: If the time required by each channel to process a target varies stochastically across trials and the distributions of processing times for the two channels overlap, then the mean time required for the first of two targets to be recognized will be less than the mean time required for either target individually. Put loosely, mean RT is decreased on redundant-target trials because there are two chances for a fast target identification, as opposed to only one chance on single-target trials.

The second class capable of explaining the RSE involves coactivation. These models allow evidence concerning the presence of at least one target to be "pooled" across channels prior to decision (e.g., Fournier \& Eriksen, 1990; Grice et al., 1984; Miller, 1982; Mordkoff \& Yantis, 1991; see also Diederich \& Colonius, 1987). This class explains the RSE in terms of energy or activation summation: With two targets contributing activation on redundant-target trials, the criterion required to trigger a response will often be reached more quickly. Recall here the saying "many hands make light work."

Because both model classes are consistent with the RSE in mean RT, more sophisticated measures have been developed to discriminate between them. These tests involve the analysis of entire distributions of RT. Two specific distribution-based tests have been used to examine the RSE: (1) the race-model inequality (Miller, 1978, 1982) and (2) the combination-rule regression analysis based on variable-criterion theory (Grice et al., 1984). ${ }^{1}$ The racemodel inequality has been discussed in detail previously (e.g., Eriksen, 1988; Miller, 1982; Miller \& Lopes, 1991; Mordkoff \& Yantis, 1991), so its application to the RSE will not be reviewed again here. The present study was concerned only with the combination-rule regression analysis.

\section{Variable-Criterion Theory and the Combination Rule}

The combination-rule regression analysis (Grice et al., 1984) is a recent extension of variable-criterion theory (Grice, 1968)-a quantitative model of perception and re- 
sponse activation that has been applied to a variety of RT tasks (for an introduction and summary, see Grice, Nullmeyer, \& Spiker, 1982). The general theory explicitly assumes that response activation builds slowly (following a deterministic function) and that the threshold required to trigger an overt response varies across trials following a normal distribution (see, e.g., Grice et al., 1984, p. 455), hence the name of the theory. These assumptions afford the possibility of calculating the mean level of response activation-referred to as "excitatory strength," or $E$, by Grice and his colleagues-at any time $t$ since stimulus onset. This is done by finding the normal deviate of the cumulative distribution function (CDF) of RT:

$$
E(t)=\Phi^{-1}[P(\mathrm{RT} \leq t)]+3.5,
$$

where $E(t)$ denotes excitatory strength at time $t$, and $\Phi^{-1}$ is the inverse of the integral of the unit normal distribution-in other words, the normal deviate. The constant of 3.5 is added to keep $E$ above zero for most values of $t$, creating a realistic origin.

The combination rule is a specific application of variable-criterion theory to redundant-target detection and the RSE. The rule entails a simple linear regression on the values of $E$ found using the equation above. Three sets of values of $E$ are required: one set for the redundanttarget condition, and one each for the two single-target conditions. The slope and intercept values from the regression describe the growth of $E$ on redundant-target trials relative to single-target trials:

$$
E\left(t \mid \mathrm{T}^{1} \& \mathrm{~T}^{2}\right)=m\left[E\left(t \mid \mathrm{T}^{1} \text { only }\right)+E\left(t \mid \mathrm{T}^{2} \text { only }\right)\right]+b,
$$

where $E\left(t \mid \mathrm{T}^{1} \& \mathrm{~T}^{2}\right)$, for example, is the value of $E$ at time $t$ given redundant targets in Display Locations 1 and 2 . The values of $t$ used are the 19 RTs corresponding to the 5th through 95th percentiles (at 5\% intervals) for the redundant-target condition. These values are selected for each subject individually, then the values of $E$ are averaged across subjects prior to the regression. This procedure is analogous to the Vincentizing performed in other distribution-based tests (e.g., the race-model inequality; Miller, 1982).

Interpretation of combination-rule values. Grice et al. (1984) have given simple interpretations of the slope $(m)$ and intercept $(b)$ values from the combination rule. This is most easily shown by example. Thus, assume that a redundant-target detection task has been analyzed using the combination rule, producing slopes and intercepts of 0.5 each, with an $r^{2}$ over 0.99 for the regression. (It should be noted that these values are typical of empirical research.) In Grice et al.'s (1984) words: “These results indicate, throughout the distribution, a clear summation effect of the separate functions, each contributing about half its strength"' (p. 456). In other words, the 0.5 slope is taken as evidence that each of the targets within a redundant-target display contributes half of the excitatory strength it would contribute on a single-target trial. This interpretation may also seem to be bolstered by the good fit (high $r^{2}$ ) provided by the regression (but for a contrary view, see various papers by N. H. Anderson, e.g., Anderson, 1962; Sidowski \& Anderson, 1967).

Now also assume that these particular values have been observed in not one, but a series of experiments. Under these conditions, the conclusion becomes much stronger:

The credibility and utility of any measurement model is primarily based on the degree to which the results provide simple and orderly descriptions of empirical relations. The results appear to be too precise and too general to be the result of a fortuitous accident. Taken as a whole, the research presents nearly conclusive evidence that redundant stimuli combine on the basis of strength rather than proportions following the rules of probability. In this respect, the results confirm the conclusions of Miller (1982) and provide evidence concerning the nature of the combination rule. (Grice et al., 1984, p. 461)

Because Miller (1982) concluded in favor of coactivation models, it seems clear that one is to reject race models given a slope value of 0.5 coupled with a large value of $r^{2}$. It is as yet unclear what combination-rule values must be observed in order to reject coactivation models. One purpose of the simulations reported next was to determine what values are consistent with each class of models. ${ }^{2}$

\section{Simulations}

Each of the simulations was based on one of two general models: an independent race model and a coactivation model (for simple schematics of these models, see Figure 1 of Mordkoff $\&$ Yantis, 1991, p. 521). ${ }^{3}$ These models are distinct only in terms of their decision mechanisms: The race model assumes separate decisions made in parallel, while the coactivation model assumes a combined decision, based on the identities of both display items. Otherwise, the models are identical; for example, encoding and response selection are the same, and both models assume a sequence of nonoverlapping stages (i.e., no partial information is transferred between stages; $\mathrm{cf}$. Miller, 1988).

Simulated race model. Under the race model, each of the two display items is first encoded by separate and independent channels. Next, separate decisions are made as to whether each item is a target. If a target is found by either channel, then a target-present code is passed forward to those processes that activate responses-the first such code triggers the response on redundant-target trials. Identified nontarget items are assumed to have no effect on decision or response selection. This is an independent race model (although it also resembles the model presented by Ulrich \& Giray, 1986).

The time required by each channel to encode a display item was simulated as a Gaussian with mean $\mu_{E}$ and variance $\sigma_{E}^{2}$. The time required by each of the decision processes was simulated using a Poisson counting process; decision time was the sum of $N$ exponentials, each with 
Table 1

Summary of the Results from the Combination-Rule Regression Analyses

\begin{tabular}{lccccccc}
\hline & \multicolumn{3}{c}{ Independent Race Models } & & \multicolumn{3}{c}{ Coactivation Models } \\
\cline { 2 - 4 } & Slope & Intercept & $r^{2}$ & & Slope & Intercept & $r^{2}$ \\
\hline$M$ & 0.53 & 0.23 & 0.994 & & 0.55 & 0.28 & 0.992 \\
$S D$ & 0.03 & 0.13 & 0.004 & & 0.04 & 0.20 & 0.007 \\
Minimum value & 0.47 & -0.13 & 0.981 & & 0.48 & -0.09 & 0.965 \\
Maximum value & 0.61 & 0.47 & 0.999 & 0.62 & 1.00 & 0.999 \\
\hline
\end{tabular}

$M$ and $S D \tau$. The time required for a response to be selected and initiated was simulated as a Gaussian with mean $\mu_{R}$ and variance $\sigma_{R}^{2}$.

On this model, single-target RT was the sum of three values, one sampled from each of these three distributions. For a redundant-target trial, the first two distributions were sampled twice each-with each pair representing the time required to encode and decide about one of the two targets-and the pair with the smaller sum (i.e., the winner of the "race") was retained. To this value, a sample from the distribution simulating response selection and initiation was added.

Simulated coactivation model. The coactivation model used for these simulations is a revised form of the superposition model presented by Schwarz (1989). As under the race model, each stimulus is encoded independentlythe difference between the two models concerns how decisions are made on redundant-target trials. In this case, both targets contribute activation to a common decision threshold on a redundant-target trial. Once criterion has been reached, a response is triggered.

Encoding was simulated in the same manner as under the race model: using a Gaussian with mean $\mu_{E}$ and variance $\sigma_{E}^{2}$. Also similar to the race model, decision time for each target was simulated using a Poisson counting process involving an exponential with $M$ and $S D \tau$. However, in contrast to the race model, the two processes described by the exponentials were both contributing to the same criterion, $N$, on redundant-target trials. Finally, as above, the time required to select and activate the response was simulated as a Gaussian with parameters $\mu_{R}$ and $\sigma_{R}$.

Parameter settings. A total of 128 different models were simulated, each differing in terms of the five variables: (1) model type, (2) $\sigma_{E}$, (3) $\tau$, (4) $N$, and (5) $\sigma_{R}$. For all of the simulations, the value of $\mu_{E}$ was fixed at $80 \mathrm{msec}$, the value of $\mu_{R}$ was fixed at 200 msec (because neither would have any effect on anything other than mean RT), and the values of $\tau$ and $N$ were always equal to each other. (Additional simulations have shown that using values of $\tau$ and $N$ that differ from each other would not cause any qualitative change in the results.) The values of $\sigma_{E}(5,15,25$, and $35 \mathrm{msec}), \sigma_{R}(5,15,25$, and $35 \mathrm{msec}$ ), and $\tau$ and $N(2,4,5$, and $6 \mathrm{msec})$ were manipulated in a $4 \times 4 \times 4$ factorial design, creating 64 models of each type. Other relevant parameters were fixed at values that mimic experimental work: the number of trials per condition per block of trials was 12 , the number of blocks per experimental session was 12 , and the number of subjects per experiment was 12 .
Results and Analysis. The results from all 128 simulations were analyzed using the combination-rule regression analysis introduced above. Summaries of the results appear in Table 1 and Figure 1. Most notable is the consistent finding of a slope near 0.5 coupled with a very high value of $r^{2}$. This pattern held true for both types of model. It is clear, therefore, that combination-rule slopes and values of $r^{2}$ cannot be used to discriminate between independent race and coactivation models.

According to the comments on interpretation given above, all of the data shown in Figure 1 would be taken as support for coactivation models as a class. Such a conclusion would be erroneous in half of these cases, because only half of these data points represent the results from a coactivation-model simulation. Thus, the finding of a 0.5 slope cannot be taken as evidence that each target within a redundant-target display is "contributing half of its strength" (Grice et al., 1984, p. 456): Under the coactivation model that was simulated, each target contributed all of its strength, while only one target (on any given trial) contributed any activation under the race model, yet approximately 0.5 slopes were observed in each case.

It should also be noted that, if anything, the fits to the data provided by the combination-rule regression were better for the independent race model (see Table 1). (A $t$ test-of somewhat questionable validity-was conducted

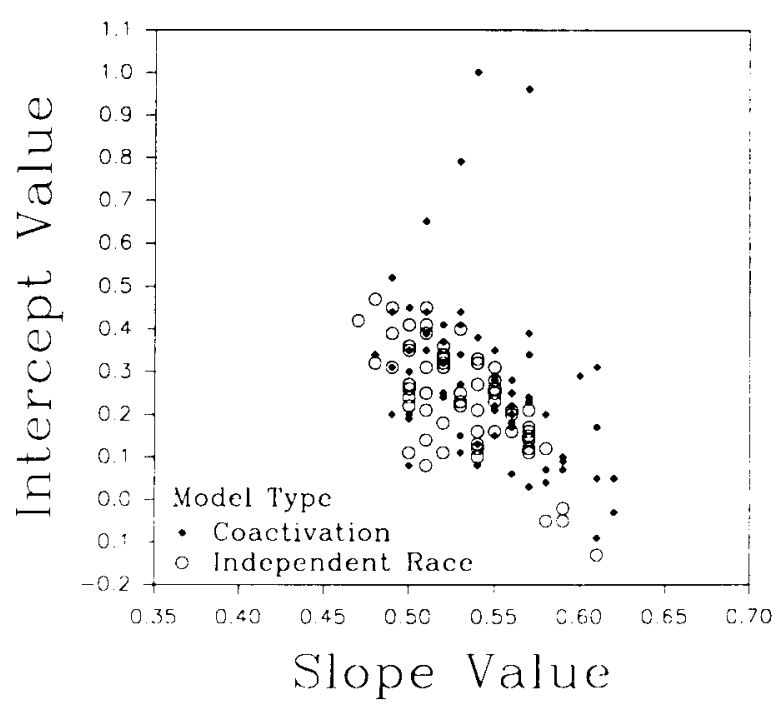

Figure 1. Combination-rule slopes and intercepts from all 128 simulations. (Note-Many data points are superimposed.) 
on the values of $r^{2}$; they were significantly higher for the race-model simulations; $p<.025$.) Thus, a high $r^{2}$ value cannot be taken as support for coactivation models. In general, the observed values of $r^{2}$ were extremely high because the three distributions that are used in each regression were very similar-all being monotonic, nondecreasing functions of $t$ derived from the CDFs of RT. ${ }^{4}$

The only difference between models in the results from the combination-rule tests concerned the intercept values. Although the mean values were similar, the coactivation model sometimes produced a relatively large intercept (see Figure 1), suggesting that this test might be sensitive to some difference between the two model classes. Further support for this claim might be taken from the finding of a positive correlation between the presence of significant violations of the race-model inequality (Miller, 1982) and the combination-rule intercept value $(r=0.51$, $p<.001)$. However, the four extreme intercept valuesthat is, those that noticeably exceed the values obtained from race-model simulations-all came from coactivationmodel simulations with low encoding- and responserelated variance (i.e., $\sigma_{E}^{2}$ was 5 and $\sigma_{R}^{2}$ was 5 or 15 ) and high decision variance (i.e., $\tau$ and $N$ were 5 or 6 ). Such parameter settings also produced violations of the racemodel inequality over a wider range than has ever been observed empirically - for example, the simulation with the largest intercept value violated the race-model inequality at all quantiles up to and including the 90 th. For this reason, the potential of using intercept values as a sign of coactivation seems limited.

Discussion. One question raised by these findings concerns why independent race and coactivation modelsalong with several types of serial model (see Note 3)produce such similar results on the combination-rule regression. In particular, why do these models produce slope values of 0.5 so consistently?

One possibility is that the use of a Gaussian to simulate predecisional processes, coupled with variable-criterion theory's use of the normal deviate to estimate excitatory strength $(E)$, has caused this result. To test this alternative, another set of independent race models were examined, these using a Poisson counting process to simulate encoding time. To keep the expected value of encoding time the same as before, pairs of $\tau$ and $N$ were chosen such that their product was 80 (the specific values were 16 and 5, 10 and 8,8 and 10, and 5 and 16); otherwise these simulations were the same as the original race model.

The results from these simulations disconfirmed the proposal that the use of a Gaussian within the present simulations was responsible for the consistent 0.5 slope. Again the slopes were always approximately 0.5 , and the values of $r^{2}$ remained at or above 0.990 .

Another possible explanation of the consistent results would cite the use of identical encoding-time distributions for each of the two targets. In the main simulations, both were Gaussian; in the ancillary simulations reported above, both were Poisson counting processes. To test this idea, another 32 simulations were conducted (16 race models and 16 coactivation models). For each of these, the encoding time of one target was simulated using a Gaussian (always with an expected mean of $80 \mathrm{msec}$ ), the other using the Poisson counting process (also with an expected mean of $80 \mathrm{msec}$ ). Again, the results were the same: a consistent 0.5 slope and high $r^{2}$ value for both types of model.

Although no principled explanation for the consistent 0.5 slope has yet been presented, it still seems important to explain why these results undermine the original conclusion of Grice et al. (1984). These researchers claimed that the 0.5 regression slopes that they observed were due to each target on a redundant-target trial contributing half of the excitatory strength it would contribute on a singletarget trial. Note that they did not claim that the total amount contributed by each target was half of the maximum; the regression is across values of time $(t)$, so the conclusion was that the buildup or rate of growth of activation strength is cut in half on redundant-target trials. However, this conclusion cannot be applied to the present data: All of the coactivation models that were simulated used the same growth-determining parameters (i.e., $\tau$ and $N$ ) on single- and redundant-target trials, and the regression still produced a slope of $0.5 .^{5}$

To explore this point further, another 48 simulations were conducted using a coactivation model that had been modified to more closely resemble that for which Grice et al. (1984) argued. (The same values of $\sigma_{E}^{2}$ and $\sigma_{R}^{2}$ were used as in the main simulations, while $\tau$ and $N$ were raised to 3, 6, or $9 \mathrm{msec}$ to give comparable RSEs.) Under this model, single-target trials were simulated in the same manner as under the "standard" coactivation model. However, on redundant-target trials, the value of $\tau$ was doubled (to 6, 12, or $18 \mathrm{msec}$, to cut in half the growth of excitatory strength for each target), and the value of $N$ was decreased by one third (to 2,4 , or $6 \mathrm{msec}$, to represent the baseline shift evidenced by the above-zero intercepts).

The results from the combination-rule regressions on these data were similar to those from the original coactivation model: As before, the slope value was always near 0.5 with an $r^{2}$ of about 0.995 , although the intercepts now ranged from -0.22 to 1.60 .

Analytic discussion. While it has become clear that the combination-rule regression analysis cannot be used to discriminate between independent race and coactivation models, one may still wish to know why 0.5 slopes are observed so consistently. To understand this, we must turn to a more analytic approach.

As a first step, recall that for any simple linear regression, for example,

$$
Y=m X+b,
$$

the following rule also applies:

$$
m=r\left(\sigma_{Y} / \sigma_{X}\right),
$$

where $m$ is the slope from the regression, $r$ is the correlation between the $Y$ and $X$ values, and $\sigma_{Y}$ and $\sigma_{X}$ are the 
standard deviations of the $Y$ and $X$ values, respectively. In the case of the combination-rule regression, the $X$ values are the sums of the two single-target $E$ values (see Equation 2), but this will not cause any change in the present analysis because a simple linear regression is still conducted.

Next, recall that for the regressions performed on the simulation data, the values of $r^{2}$ were all near 1.00 (due to the use of CDFs; see Note 4); thus, the value of $r$ may be removed from Equation 4 and the slope must be equal to the ratio of the standard deviations. If we replace the $Y$ in Equation 4 with the symbol $E R$, for $E$ given redundant targets, and the $X$ with $E S 1+E S 2$, for $E$ given Target 1 (only) plus $E$ given Target 2 (only), then we arrive at:

$$
m=\sigma_{E R} / \sigma_{E S 1+E S 2}
$$

The value $\sigma_{E S 1+E S 2}$ (i.e., the standard deviation of the sum of $E S 1$ and ES2) may be expanded using the following:

$$
\sigma_{E S 1+E S 2}^{2}=\sigma_{E S 1}^{2}+\sigma_{E S 2}^{2}+2 \sigma_{E S 1, E S 2},
$$

where $\sigma_{E S 1, E S 2}$ is the covariance between the $E S 1$ and $E S 2$ values. From another formula involving covariance, that is,

$$
\sigma_{E S 1, E S 2}=r \sigma_{E S 1} \sigma_{E S 2},
$$

and the fact that the correlation between two cumulative distributions is necessarily very high (see also Table 2)which allows for the removal of the term $r$ from Equation 7-we achieve:

$$
m=\sigma_{E R} /\left(\sigma_{E S 1}^{2}+\sigma_{E S 2}^{2}+2 \sigma_{E S 1} \sigma_{E S 2}\right)^{1 / 2} .
$$

After some reduction in terms, we arrive at a simple formula for the expected slope value:

$$
m=\sigma_{E R} /\left(\sigma_{E S 1}+\sigma_{E S 2}\right) \text {. }
$$

Equation 9 should begin to make clear why slopes near 0.5 are observed so frequently. Yet, we may clarify this point even further. To this end, note that the standard deviation of the entire distribution of $E R$ values (i.e., $\sigma_{E R}$ ) is necessarily 1.00 because the $E R$ values are the unit normal deviates of the redundant-targets CDF plus a constant (Equation 1). However, the only values of $E R$ used in the combination-rule regression are those that correspond to

Table 2

Summary Statistics (Standard Deviations, Covariance, and Correlation) Concerning the Excitatory-Strength Functions

\begin{tabular}{lccccc}
\hline & \multicolumn{2}{c}{ Independent Race Models } & & \multicolumn{2}{c}{ Coactivation Models } \\
\cline { 2 - 3 } \cline { 5 - 6 } & $M$ & $S D$ & & $M$ & $S D$ \\
\hline$\sigma_{E R}$ & 0.87 & 0.00 & & 0.87 & 0.00 \\
$\sigma_{E S 1}$ & 0.82 & 0.05 & & 0.79 & 0.06 \\
$\sigma_{E S 2}$ & 0.81 & 0.05 & & 0.80 & 0.05 \\
$\sigma_{E S 1+E S 2}$ & 1.63 & 0.09 & & 1.59 & 0.11 \\
$\sigma_{E S 1, E S 2}$ & 0.67 & 0.08 & & 0.63 & 0.08 \\
$r_{E S 1, E S 2}$ & 0.998 & 0.002 & & 0.998 & 0.002 \\
\hline
\end{tabular}

the 5 th-95th percentiles at $5 \%$ intervals, and these 19 values will always have a standard deviation of only 0.87 , instead (see Table 2). Similarly, the ES1 and ES2 values are the unit normal deviates of the single-target CDFs (plus a constant), also evaluated at only 19 points; however, the full range of these values will not enter into the regression because $E S 1$ and $E S 2$ are evaluated at the RT points taken from the redundant-targets condition. This will often truncate the $E S 1$ and $E S 2$ distributions (in particular, the "slow tail" from each will be removed), slightly reducing both $\sigma_{E S 1}$ and $\sigma_{E S 2}$. Therefore, a slope slightly above, but quite near, 0.5 will be often observed. Slopes below 0.5 are also possible, but should be less frequent. This occurs when the truncation of the single-target distributions actually increases the mean of $\sigma_{E S 1}$ and $\sigma_{E S 2}$ to being above 0.87 .

That the expected slope value is slightly above 0.5 has importance with regard to the excitatory-strength origin. Recall that 3.5 is added to all normal-deviate scores to derive the values of $E$ (Equation 1). On the proviso that all values of $E$ remain above zero, any change to the specific constant selected will have no effect on the slope value, nor will it affect the value of $r^{2}$. However, the combination-rule intercept will be affected as follows:

$$
\Delta b=(1-2 m) \Delta c,
$$

where $\Delta b$ is the change in intercept value, $m$ is the slope, and $\Delta c$ is the change in the selected additive constant for Equation 1 . As can be seen, if the slope differs from 0.5 , the intercept will depend on the specific additive constant. This point has been verified by reanalysis of the original 64 race-model simulations using a constant of 7.0 in Equation 1 (rather than 3.5): Consistent with Equation 10 and the finding of a mean slope of 0.53 , the combinationrule intercepts now had a mean of only 0.01 , as contrasted with 0.23 (see Table 1).

\section{Conclusion}

The combination-rule regression analysis (Grice et al., 1984) cannot be used to discriminate between the various parallel-processing models that are capable of explaining the redundant-signals effect. Furthermore, no arguments concerning psychological mechanisms should be based on the regression slope values, even when a high value of $r^{2}$ is found. Nearly identical results may be produced by members of both competing model classes; in fact, the major finding from previous use of combination rule-a slope near 0.5-appears to be a tautology due to the use of cumulative distributions.

\section{REFERENCES}

ANDERSON, N. H. (1962). Application of an additive model to impression formation. Science, 138, 817-818.

Biederman, I., Checkosky, S. F. (1970). Processing redundant information. Journal of Experimental Psychology, 83, 486-490.

CoLonius, H. (1990). Possibly dependent probability summation of reaction time. Journal of Mathematical Psychology, 34, 253-275.

Diederich, A., CoLonius, H. (1987). Intersensory facilitation in the motor component? Psychological Research, 49, 23-29. 
Egeth, H. E. (1966). Parallel versus serial processes in multidimensional stimulus discrimination. Perception \& Psychophysics, 1 , 245-252.

EGETH, H. E., MoRDKoFf, J. T. (1991). Redundancy gain revisited: Evidence of parallel processing of color and form. In J. Pomerantz \& G. Lockhead (Eds.), The perception of structure (pp. 131-143). Washington, DC: American Psychological Association.

ERuksen, C. W. (1988). A source of error in attempts to distinguish coactivation from separate activation in the perception of redundant targets. Perception \& Psychophysics, 44, 191-193.

Fournier, L. R., ERIKSEN, C. W. (1990). Coactivation in the perception of redundant targets. Journal of Experimental Psychology: Human Perception \& Performance, 16, 538-550.

GRICE, G. R. (1968). Stimulus intensity and response evocation. Psychological Review, 75, 359-373.

Grice, G. R., Canham, L., Boroughs, J. M. (1984). Combination rule for redundant information in reaction time tasks with divided attention. Perception \& Psychophysics, 35, 451-463.

GrJce, G. R., Nullmeyer, R., \& SPIKER, V. A. (1982). Human reaction time: Toward a general theory. Joumal of Experimental Psychology: General, 111, 135-153.

Holmgren, J. L., Joula, J. F., \& Atkinson, R. C. (1974). Response latency in visual search with redundancy in the visual display. Perception \& Psychophysics, 16, 123-128.

MilleR, J. (1978). Multidimensional same-different judgments: Evidence against independent comparisons of dimensions. Journal of Experimental Psychology: Human Perception \& Performance, 4, 411-422.

Miller, J. (1982). Divided attention: Evidence for coactivation with redundant signals. Cognitive Psychology, 14, 247-279.

MiLleR, J. (1988). Discrete versus continuous stage models of human information processing: In search of partial output. Acta Psychologica, 67, 1-67.

MILLER, J., LoPES, A. (1988). Testing race models by estimating the smaller of two true mean or true median reaction times: An analysis of estimation bias. Perception \& Psychophysics, 44, 513-524.

Miller, J., Lopes, A. (1991). Bias produced by fast guessing in distribution-based tests of race models. Perception \& Psychophysics, 50, 584-590.

Mordkoff, J. T., \& YANTIS, S. (1991). An interactive race model of divided attention. Journal of Experimental Psychology: Human Perception \& Performance, 17, 520-538.

Mulligan, R. M., Shaw, M. L. (1980). Multimodal signal detection: Independent decisions vs. integration. Perception \& Psychophysics, 28, 471-478.

Mulun, P. A., Egeth, H. E., Mordkoff, J. T. (1988). Redundanttarget detection and processing capacity: The problem of positional preferences. Perception \& Psychophysics, 43, 607-610.

RAAB, D. (1962). Statistical facilitation of simple reaction time. Transactions of the New York Academy of Sciences, 43, 574-590.

SCHWARz, W. (1989). A new model to explain the redundant-signals effect. Perception \& Psychophysics, 46, 498-500.

SHAw, M. L. (1982). Attending to multiple sources of information: I. The integration of information in decision making. Cognitive Psychology, 14, 353-409.

SHAW, M. L. (1984). Division of attention among spatial location: A fundamental difference between detection of letters and detection of luminance increments. In H. Bouma \& D. G. Bouwhuis (Eds.), Attention and performance (Vol. 10, pp. 106-121). Hillsdale, NJ: Erlbaum.
Sidowski, J. B., Anderson, N. H. (1967). Judgments of CityOccupation combinations. Psychonomic Science, 7, 279-280.

UlRICH, R., \& GRAY, M. (1986). Separate-activation models with variable base times: Testability and checking of cross-channel dependency. Perception \& Psychophysics, 39, 248-254.

van der Hejuden, A. H. C. (1975). Some evidence for a limited capacity parallel self-terminating process in simple visual search tasks. Acta Psychologica, 39, 21-41.

van der Hejden, A. H. C., LA HeiJ, W., \& Boer, J. P. A. (1983). Parallel processing of redundant targets in simple search tasks. Psychological Research, 45, 235-254.

van der Heijden, A. H. C., Schreuder, R., Maris, L., \& NeeRINCX, M. (1984). Some evidence for correlated separate activations in a simple letter-detection task. Perception \& Psychophysics, 36, $577-585$.

\section{NOTES}

1. A third test based upon the work of M. L. Shaw (e.g., Mulligan \& Shaw, 1980; Shaw, 1982, 1984) has also been used by Grice et al. (1984) and by Mordkoff and Yantis (1991). This test is very similar to that using the race-model inequality, although it requires additional assumptions.

2. It is important to note that the present study concerns only the combination-rule regression analysis and the interpretation of its results. This work does not speak to variable-criterion theory as a whole, because the combination rule is not assumed, nor can it be directly deduced from the theory (G. R. Grice, personal communication, March 1990).

3. Actually, three other models were also initially simulated: (1) an "unguided" serial-decisions model, (2) a "guided" serial-decisions model, and (3) a fixed-order, serial-decisions model. The slope and $r^{2}$ values from these models were very similar to those from the independent race and coactivation models. Summaries of these results are available from the author, as are summaries of the other simulations referred to in the text.

4. A reviewer provided a simple demonstration of how the use of cumulative distributions inflates the observed $r^{2}$ values. Consider the following two distributions that have a correlation of -1.00 :

$$
x_{1}=1, x_{2}=2, x_{3}=3, \quad x_{4}=4, \quad x_{5}=5,
$$

and

$$
Y_{1}=5, \quad Y_{2}=4, \quad Y_{3}=3, \quad Y_{4}=2, \quad Y_{5}=1 .
$$

Upon cumulation, that is,

$$
X_{1 c}=1, X_{2 c}=3, X_{3 c}=6, \quad X_{4 c}=10, X_{5 c}=15,
$$

and

$$
Y_{1 c}=5, Y_{2 c}=9, Y_{3 c}=12, Y_{4 c}=14, Y_{5 c}=15 \text {, }
$$

the correlation becomes 0.92 .

5. Grice et al. (1984) also asserted that the redundant-signals effect is due to the additive constant that raises the value of $E$ on redundanttarget trials-that is, the combination-rule intercept. However, in the present simulations, the magnitude of the RSE was uncorrelated with the observed intercept value for both independent race $(r=0.13)$ and coactivation models $(r=-0.05)$.

(Manuscript received February 6, 1992; revision accepted for publication May 20, 1992.) 